\title{
To Enhancement in Zang and Sang Thinning Algorithm to Improve Thinning Rate using Fuzzy Logic
}

\author{
Ravaljodh Singh \\ CSE, SVIET Ramnagar, \\ Banur (Punjab)
}

\author{
Lalita Bhutani \\ Head of department of SVIET \\ Banur(Punjab)
}

\begin{abstract}
The main concept in this paper is that to produce clear pixels of from an image which can be shown clearly. Many methods have been introduced in this area, but in this paper a new method has been proposed which is worked on the basis of skeletonization. In this method different parameters have been used and these are the performance evaluation of proposed technique in comparison with existing algorithms such as execution time, thinning rate, number of connected components, PSNR, MSE etc.
\end{abstract}

\section{Keywords}

Skeletonization, Thinning of the image, PSNR and MSE.

\section{INTRODUCTION}

Skeletonization is an important step in many image processing applications like fingerprint analysis [4], optical character recognition [2,5], medical imaging [4]. Therefore, it is an active area of research. There is always a need for good skeletonization algorithms in reference to following parameters:

1. To reduce the amount of data required to be processed as it takes less time [4].

2. To reduce processing time.

3. Extraction of critical features such as end-points, junction-points, and connection among the components is helpful in many applications [4].

4. By reducing an object to only a skeleton, unimportant features and image noise can be filtered out.

5. Skeletonization is commonly used in the higher degree analysis and recognition for applications such as diagram understanding, OCR [2.5], feature detection, and fingerprint analysis [4].

But most of the skeletonization algorithms suffer from traditional problems such as reducing to one pixel width of the skeleton, preserving geometrical and topological properties. Many of the algorithms have the problem of discontinuity in the images. Whereas, several techniques are failed to preserve the shape topology and not reconstruct able. Spurious tails and rotating the text shape is other serious problem and due to this most of the thinning methods are failed.

Thinning problem requires two tasks to be implemented: (a) peeling the thick pixels off (b) stopping the peeling process when the pixel size reduces to exactly one. The first can be achieved with relative ease.

The main difficulty arises in the second part, because the stopping decision must be done automatically. This can be achieved using a real time cellular neural network by training the neural network. Most of the conventional thinning approaches suffer from noise sensitivity and rotation dependency. With the use of neural networks we can perform thinning invariant under arbitrary rotations. [8]

Most of the neural network training has been done on recognition different language characters, pattern classification, etc. Less work has been done on the neural network training to perform skeletonization operations. Neural network takes a different approach as compare to conventional thinning. Conventional thinning is based on algorithmic approach i.e. computer follows a specific set of instructions or conditions in order to delete the unwanted pixels. Unless the specific steps that the computer needs to follow are known the computer cannot delete the pixels. That restricts the problem solving capability in conventional thinning. But neural network process information as the human brain does. Neural networks learn by example. They can be trained to perform thinning operations effectively. They reduce the number of instructions to be executed. Hence they take less execution time and faster than the conventional thinning approaches.

An important aspect of skeletonization algorithm is noise immunity. With the use of neural networks we can handle both types of noise in the images i.e. boundary noise and object noise and make the algorithms more robust which is not possible with conventional thinning techniques. [9]

\section{PROPOSED ALGORITHM}

The main steps are described below:

1. Create a dataset of Gurumukhi script.

2. Implementing existing algorithms: To implement the existing skeletonization algorithms using neural networks.

3. Proposing a new Method for skeletonization using neural networks.

4. Evaluating Performance: To evaluate the performance of existing algorithms and new proposed method for skeletonization using neural networks on the basis of some performance measures:

a. Execution Time: The time taken to obtain the output skeletons for a particular image.

b. Thinning Rate: The degree to which an object is said to be thinned or completely thinned can be measured in terms of thinning rate.

\section{METHODOLOGY}

In methodology flow chart different parameters are used for the processing of the image. These are designed as the followings. As in the first step input of image is given and initially image is converted into grey image as this image is collaborated with the training sample through which input, hidden and output layers are selected. At the second last stage binarized image is joined with trained neural, adjusted and 
biased weights and at the end thinned image is got as output and the unprocessed image is again processed.

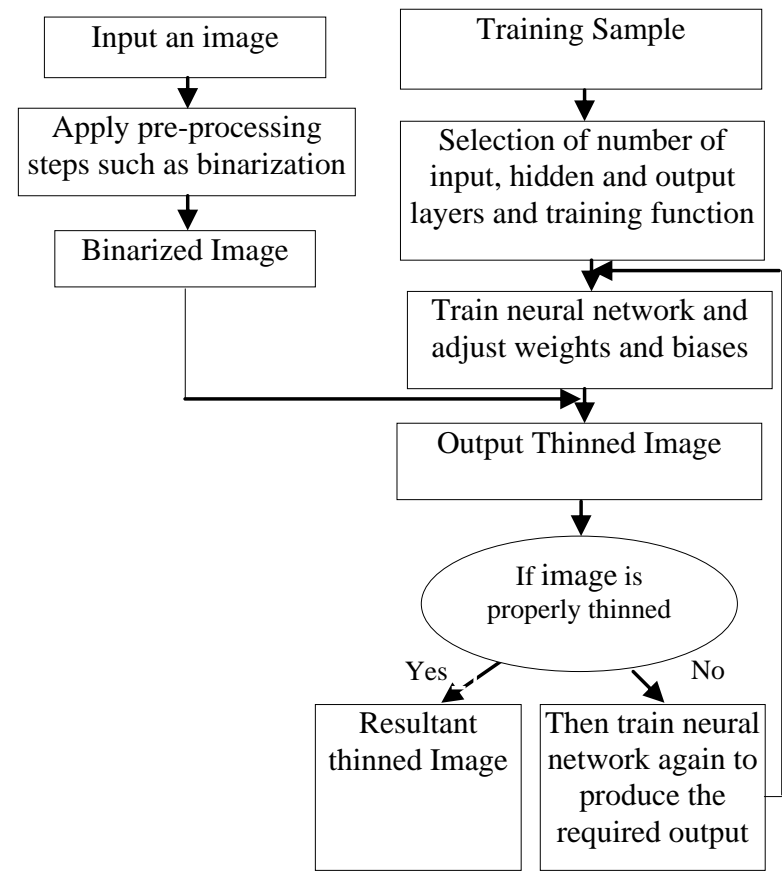

Figure 9. Proposed Methodology

\section{SKELETONIZATION}

Skeletonization is an important step in many image processing applications like Pattern recognition [2], optical character recognition [2,5], and fingerprint classification [4] etc.

1. To reduce the amount of data required to be processed as it takes less time [4].

2. To reduce processing time.

3. Extraction of critical features such as end-points, junction-points, and connection among the components is helpful in many applications [4].

4. By reducing an object to only a skeleton, unimportant features and image noise can be filtered out.

5. Skeletonization is commonly used for the higher degree analysis and recognition for applications such as diagram understanding, Optical character recognition (OCR)[2,5] , feature detection, and fingerprint analysis [4].

\subsection{Applications of Skeletonization}

Skeletonization has been used for variety of image processing applications like:
1. Optical character recognition (OCR) $[2,5]$
2. Pattern recognition[3]
3. Fingerprint classification[4]
4. Biometric authentication[5]
5. Signature verification[5]
6. Medical imaging[4]

\subsection{Introduction to Neural networks}

Neural networks are composed of simple elements called as neurons operating in parallel. A simple neuron is as shown in figure 9. These neural networks approach are inspired by the biological human nervous systems. Neural networks learn by example. They cannot be programmed to perform a specific task.

Commonly neural networks are adjusted, or trained, so that a particular input leads to a specific target output. We can train a neural network to perform a particular function by adjusting the values of the connections (weights) between elements. Such a situation is shown below in Fig 2. There, the network is adjusted, based on a comparison of the output and the target, until the network output matches the target. Typically many such input/target pairs are used, in this supervised learning, to train a network [7].

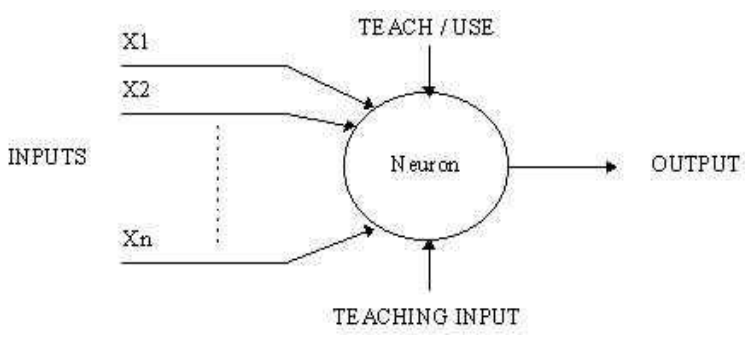

Figure 4.1: A simple neuron [6]

Figure 4.1 shows the input is entered into the neural connection and output is compared with the target value after that adjusted weights are again entered into the neural network connection.

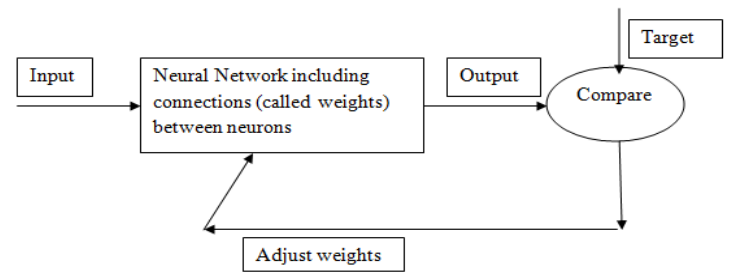

Fig 4.2. Scenario of neural networks [7]

\subsection{MODEL OF A NEURON:}

A neuron is an information-processing unit which is fundamental to the operation of the neural. The three basic elements of the neural models are:

- Synaptic Weights

- Linear Combiner

- Activation function

The brief study of these elements is given below:

- Synaptic Weights: A set of synapse which is characterized by the strength or weight of its own. It is also known as connecting links.

- Linear combiner: An adder for summing the inputs signals, weighted by the respective synapse of the neuron.

- An activation function for limiting the amplitude of output of the neuron. Sometimes it is also called squashing functions [24].

Figure 4.3 defines the four inputs which entered with the entrance of wk gate and passed through the bias gate and activation function $\mathrm{y}$ is introduced. 


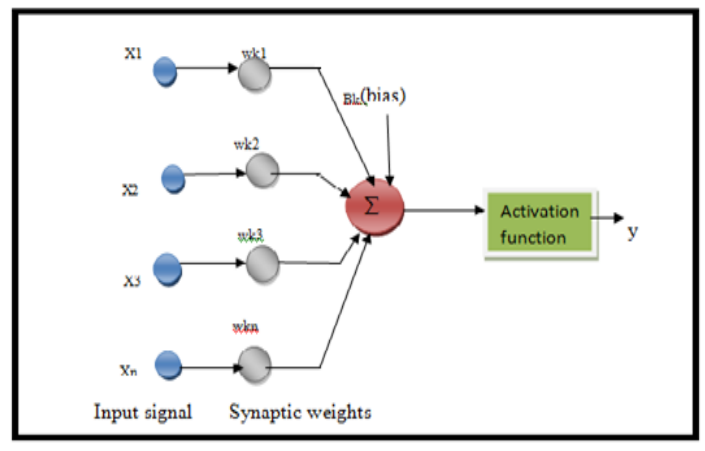

Figure 4.3 : Non Linear Model of Neuron

\subsection{NETWORK ARCHITECTURE}

The manner in which the neuron of a neural network is structured is intimately linked with the learning algorithm to train the network. So learning algorithm is used in the design of neural network as structured. Learning algorithms are used to train neural network. In general there are different classes of network architectures:

- $\quad$ Single Layer Feed Forward Networks

- Multilayer Feed Forward Networks

\subsubsection{Single Layer Feed Forward Networks:}

Figure 4.4 shows the single layer feed forward network. In a layered network the neurons are organized in the forms of layers. Input layer have source nodes that corresponds to that gives output but vice versa is not true. It is also called acyclic or Feed Forward type. Here no hidden layer is present. It is called single layer feed forward networks because it has number of input layer but only one output layer. We do not count the source node in this type of architecture because no computation is performed in this type of architecture.

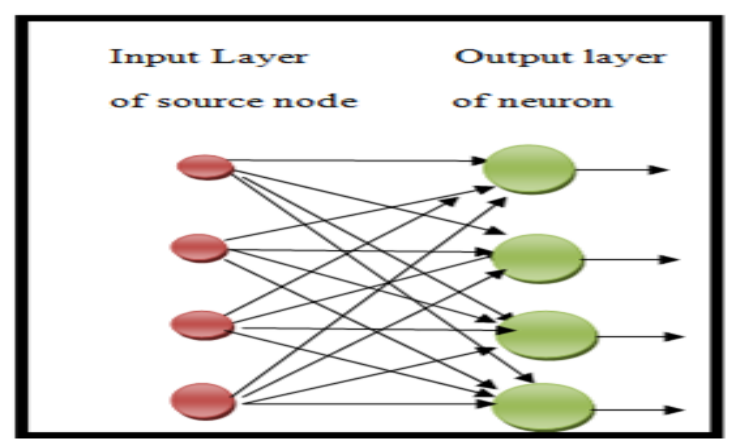

Figure 4.4: Feed Forward or acyclic network with a single layer of neuron

\subsubsection{Multilayer Feed forward Networks:}

In the second layer of feed forward networks one or more hidden layers are present, whose computation nodes are corresponding called hidden neurons and hidden units. Higher order statistics can be obtained by adding or more hidden layers. Here source nodes are called input layer, signals are applied to the computation node in the second layer.

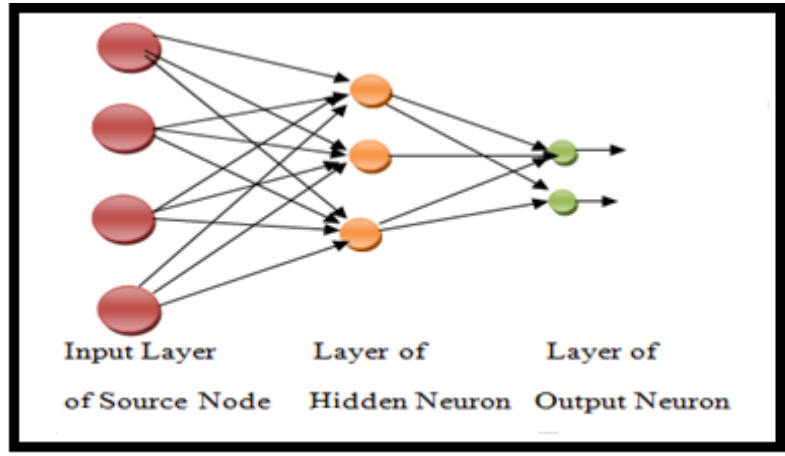

Figure 4.5: Multi connected feed forward network with one hidden layer

\section{SIMULATIONS AND RESULTS}

\subsection{Results}

In this section snapshot of results has been discussed as there are various stages which are shown as of the followings:

As illustrated in figure 5.1, the image is loaded with for the thinning. The thinning is technique of removing the unwanted data from image. To remove the unwanted data from the image thinning elements is used which will remove unwanted data. The zang and sang algorithm is used for thinning which gave output in terms of MSE, PSNR and thinning rate. The value of MSE is 6856.76, value of PSNR is 9.80 and value of TR is 0.50

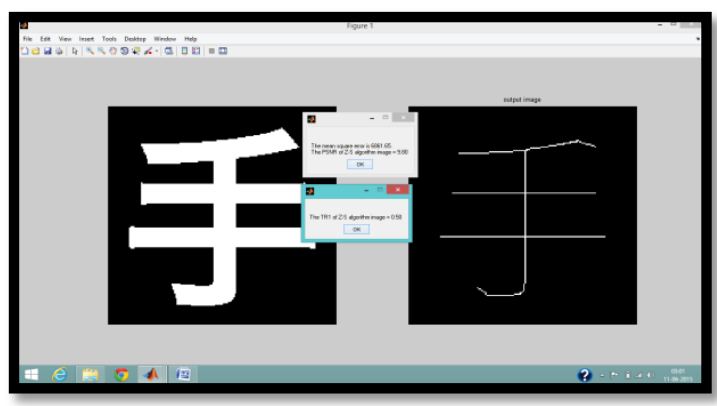

Figure 5.1 Image loaded

As shown in figure 5.2, to improve output of zang and sang algorithm in terms of PNSR, MSE and TR enhancement is proposed which will be based on back propagation algorithm. In this figure back propagation algorithm is executed with zang and sang algorithm

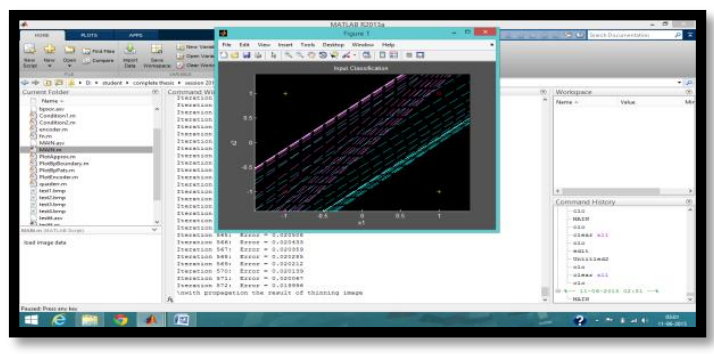

Figure 5.2 Apply of back propagation algorithm 


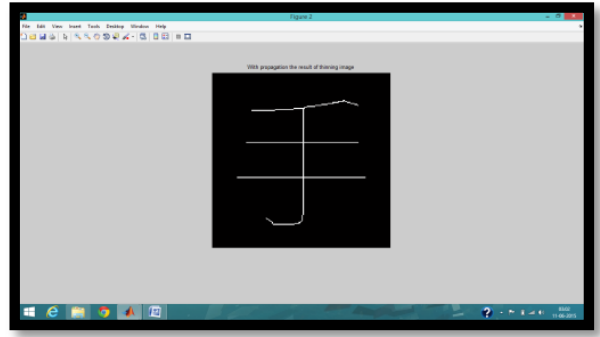

Figure 5.3: Apply of back propagation algorithm

As shown in figure 5.3, to improve output of zang and sang algorithm in terms of PNSR, MSE and TR enhancement is proposed which will be based on back propagation algorithm. In this figure back propagation algorithm is executed with zang and sang algorithm. After applying the back propagation algorithm the output of the thinning is image is shown which has better results than existing one.

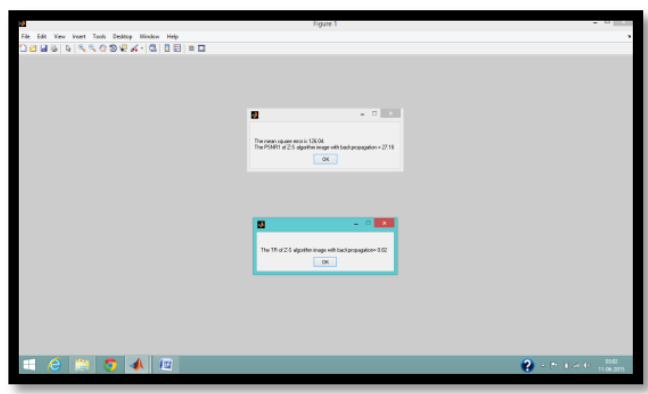

Figure 5.4: Apply of back propagation algorithm

As shown in figure 5.4, to improve output of zang and sang algorithm in terms of PNSR, MSE and TR enhancement is proposed which will be based on back propagation algorithm. In this figure back propagation algorithm is executed with zang and sang algorithm. After applying the back propagation algorithm the output of the thinning is image is shown which has better results than existing one. The results after applying MSE, PNSR and TR values are 125.91,27.16 and 0.82 respectively

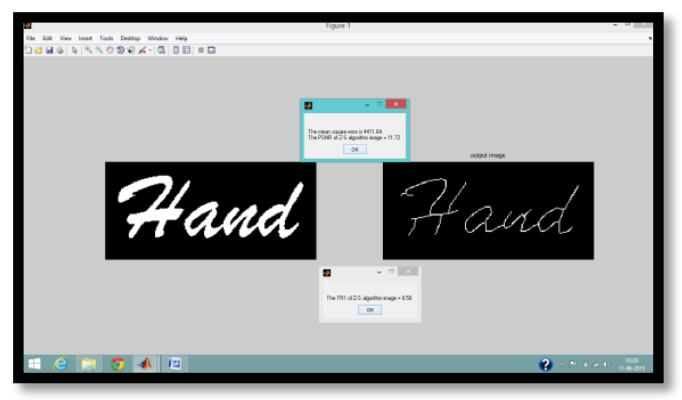

Figure 5.5 Image loaded

As illustrated in figure 5.5, the image is loaded with for the thinning. The thinning is technique of removing the unwanted data from image. To remove the unwanted data from the image thinning elements is used which will remove unwanted data. The zang and sang algorithm is used for thinning which gave output in terms of MSE, PSNR and thinning rate. The value of MSE is 4411.20, value of PSNR is 11.72 and value of TR is 0.50

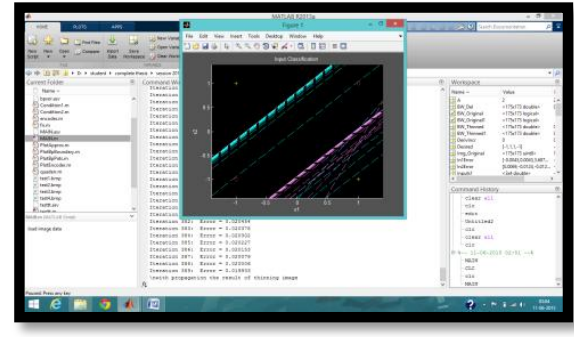

Figure 5.6 Apply of back propagation algorithm

As shown in figure 5.6, to improve output of zang and sang algorithm in terms of PNSR, MSE and TR enhancement is proposed which will be based on back propagation algorithm. In this figure back propagation algorithm is executed with zang and sang algorithm

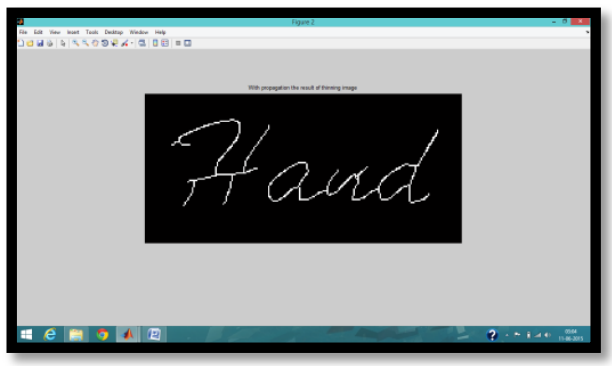

Figure 5.7 Apply of back propagation algorithm

As shown in figure 5.7, to improve output of zang and sang algorithm in terms of PNSR, MSE and TR enhancement is proposed which will be based on back propagation algorithm. In this figure back propagation algorithm is executed with zang and sang algorithm. After applying the back propagation algorithm the output of the thinning is image is shown which has better results than existing one

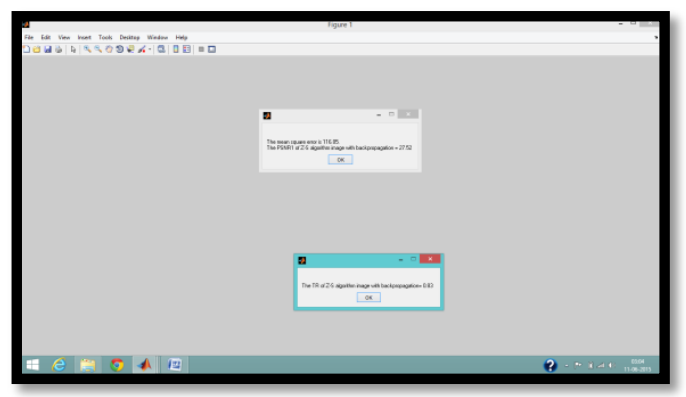

Figure 5.8 Apply of back propagation algorithm

As shown in figure 5.8, to improve output of zang and sang algorithm in terms of PNSR, MSE and TR enhancement is proposed which will be based on back propagation algorithm. In this figure back propagation algorithm is executed with zang and sang algorithm. After applying the back propagation algorithm the output of the thinning is image is shown which has better results than existing one. The results after applying MSE, PNSR and TR values are 113.32,27.16 and 0.83 respectively 


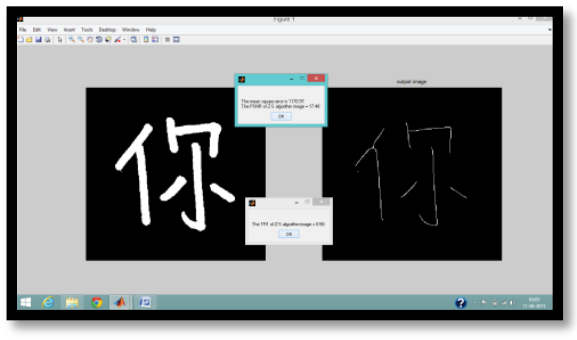

Figure 5.9 Image loaded

As illustrated in figure 5.9, the image is loaded with for the thinning. The thinning is technique of removing the unwanted data from image. To remove the unwanted data from the image thinning elements is used which will remove unwanted data. The zang and sang algorithm is used for thinning which gave output in terms of MSE, PSNR and thinning rate. The value of MSE is 1172.12, value of PSNR is 17.48 and value of $\mathrm{TR}$ is 0.50

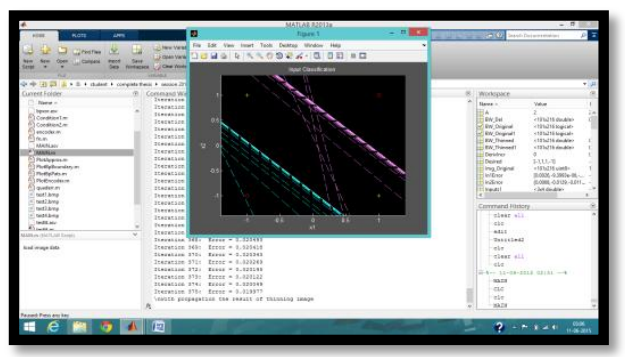

Figure 5.10 Apply of back propagation algorithm

As shown in figure 5.10, to improve output of zang and sang algorithm in terms of PNSR, MSE and TR enhancement is proposed which will be based on back propagation algorithm. In this figure back propagation algorithm is executed with zang and sang algorithm

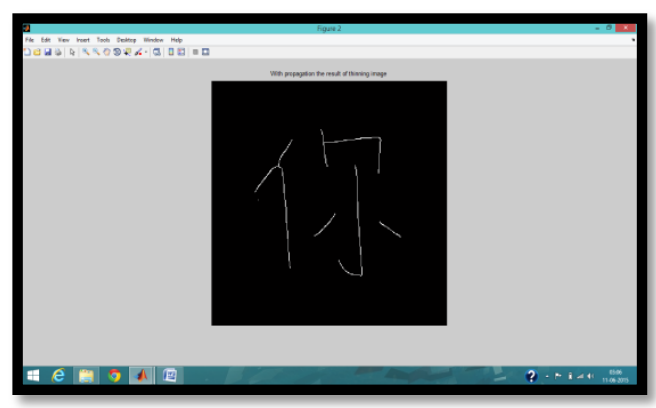

Figure 5.11 Apply of back propagation algorithm

As shown in figure 5.11, to improve output of zang and sang algorithm in terms of PNSR, MSE and TR enhancement is proposed which will be based on back propagation algorithm. In this figure back propagation algorithm is executed with zang and sang algorithm. After applying the back propagation algorithm the output of the thinning is image is shown which has better results than esxisting one

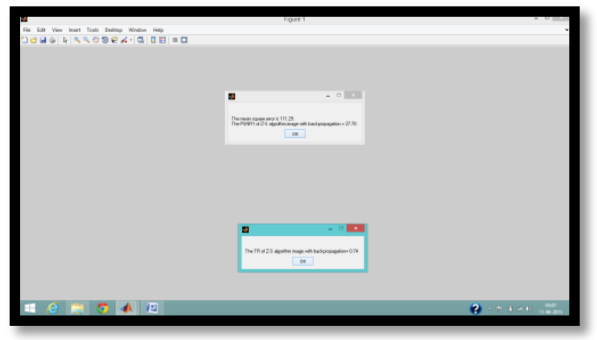

Figure 5.12 Apply of back propagation algorithm

As shown in figure 5.12, to improve output of zang and sang algorithm in terms of PNSR, MSE and TR enhancement is proposed which will be based on back propagation algorithm. In this figure back propagation algorithm is executed with zang and sang algorithm. After applying the back propagation algorithm the output of the thinning is image is shown which has better results than existing one. The results after applying MSE, PNSR and TR values are 111.57,27.69 and 0.74 respectively

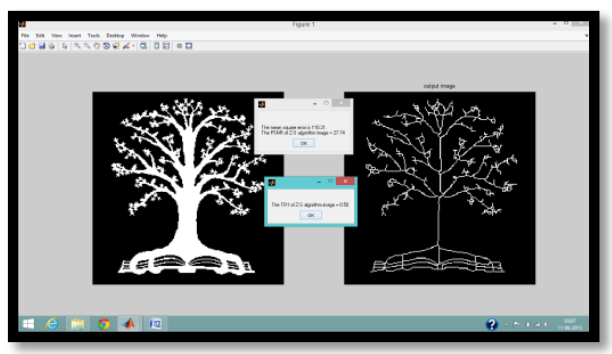

Figure 5.13 Image loaded

As illustrated in figure 5.13, the image is loaded with for the thinning. The thinning is technique of removing the unwanted data from image. To remove the unwanted data from the image thinning elements is used which will remove unwanted data. The zang and sang algorithm is used for thinning which gave output in terms of MSE, PSNR and thinning rate. The value of MSE is 109.25 , value of PSNR is 27.78 and value of TR is 0.50

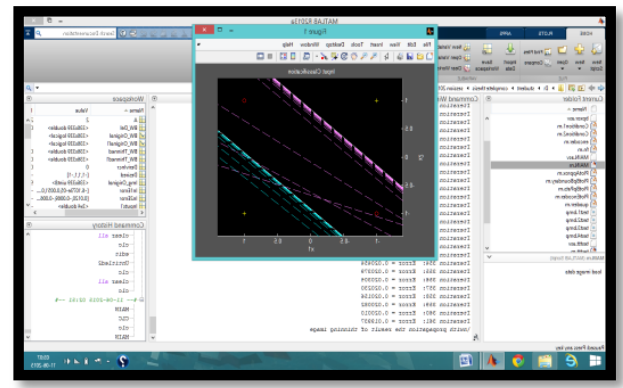

Figure 5.14 Apply of back propagation algorithm

As shown in figure 5.14, to improve output of zang and sang algorithm in terms of PNSR, MSE and TR enhancement is proposed which will be based on back propagation algorithm. In this figure back propagation algorithm is executed with zang and sang algorithm. 


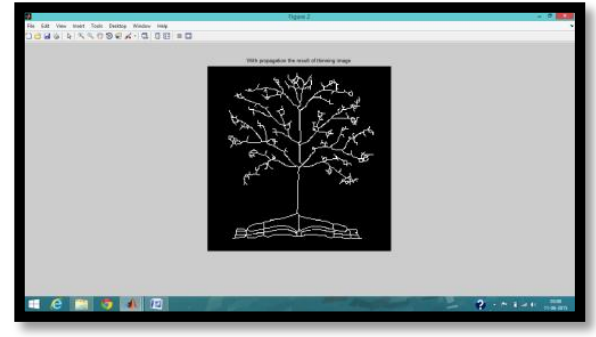

Figure 5.15 Apply of back propagation algorithm

As shown in figure 5.15, to improve output of zang and sang algorithm in terms of PNSR, MSE and TR enhancement is proposed which will be based on back propagation algorithm. In this figure back propagation algorithm is executed with zang and sang algorithm. After applying the back propagation algorithm the output of the thinning is image is shown which has better results than existing one .

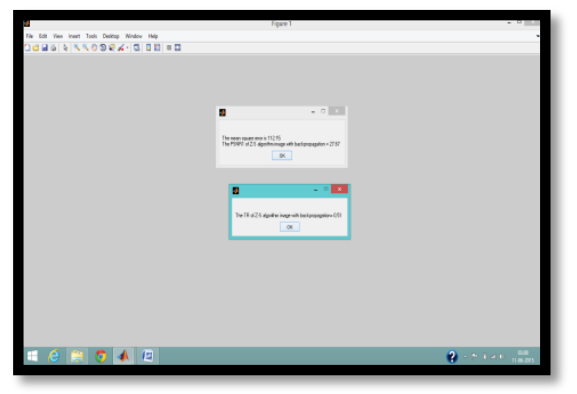

Figure 5.16 Apply of back propagation algorithm

As shown in figure 5.16, to improve output of zang and sang algorithm in terms of PNSR, MSE and TR enhancement is proposed which will be based on back propagation algorithm. In this figure back propagation algorithm is executed with zang and sang algorithm. After applying the back propagation algorithm the output of the thinning is image is shown which has better results than existing one. The results after applying MSE, PNSR and TR values are 110.87,23.72 and 0.51 respectively.

\section{CONCLUSION AND FUTURE WORK}

The thinning is techniques which can remove unwanted pixels from the images. This technique is generally used to reduce the size of image. The technique of thinning is used over the compression because it cannot remove the wanted data from the image. In this work, zang and cwang algorithm for thinning has been reviewed and implemented. To apply thinning on the images, thinning elements has been selected which have white and black pixels. The white spaces which arise on the images are removed after combining with the thinning elements. The thinning algorithm's performance has been analyzed in terms of PSNR value, MSE value thinning rate. To improve performance of the algorithm enhancement has been proposed which is based on back propagation algorithm. The simulation results shows that proposed algorithm performs batter in terms of PSNR, MSE and thinning rate. In future work, further improvement will be proposed in the enhancement and this enhancement will be based on boltzman learning. The technique of boltzman learning will improve thinning rate, PSNR and MSE value.

\section{REFERENCES}

[1] Gonzalez R.C. and Woods R.E. (2002) Digital Image Processing 2nd Ed. Tom Robbins.

[2] Abu-Ain W, et al. "Skeletonization Algorithm for Binary Images" The 4th International Conference on Electrical Engineering and Informatics (ICEEI 2013) pp.704-709.

[3] Padole G.V, Pokle S. B. "New Iterative Algorithms For Thinning Binary Images" Third International Conference on Emerging Trends in Engineering and Technology IEEE 2010 pp. 166-171

[4] Lam L, et al. " Thinning methodologies-A comprehensive survey" IEEE transactions on pattern analysis and machine intelligence Vol. 14 No. 9 September 1992 pp. 869-885

[5] Chatbri et al. " Using scale space filtering to make thinning algorithms robust against noise in sketch images" Pattern Recognition letters 42( 2014) pp. 1-10

[6] Sharma V. et al. " A comprehensive study of artificial neural networks" International journal of advanced research in computer science and computer engineering 2012 Volume 2, Issue 10 pp. 278-284

[7] Demuth H et al. Neural Network Toolbox For Use with MATLAB Mathworks july 2002

[8] Chua, L.O. ; Yokohama, T. "Image thinning with a cellular neural network." Circuits and Systems, IEEE Transactions on (Volume:37, Issue: 5 ) 1990 pp. 638640

[9] Datta A. et al "Shape Extraction: A Comparative Study Between Neural Network-Based and Conventional Techniques" Neural Computing \& Applications (1998) Springer pp. 343-355

[10] T. Zhang et al. "A fast parallel algorithm for thinning digital patterns”, Commun. ACM 27 (3) (1984) pp. 236239

[11] Zhou R.W., et al. "A novel single-pass thinning algorithm and an effective set of performance criteria" 1995 Elsevier Science pp. 1267-1275.

[12] Ahmed et al. "A Rotation Invariant Rule-Based Thinning Algorithm for Character Recognition" IEEE transactions on pattern analysis and machine intelligence, vol. 24, no. 12, December 2002 pp. 1672-1678

[13] Rockett “An Improved Rotation-Invariant Thinning Algorithm" IEEE transactions on pattern analysis and machine intelligence, vol. 27, no. 10, October 2005 pp.1671-1674

[14] Saeed K, et al. "K3M: A universal algorithm for image skeletonization and a review of thinning techniques" International Journal of Applied Mathematics \& Computer Science, 2010, Vol. 20, No. 2, pp. 317-335

[15] Jagna A. and Kamakshiprasad V. "New parallel binary image thinning algorithm" ARPN Journal of Engineering and Applied sciences vol. 5, no. 4, April 2010 pp. 64-67 